\title{
Thermal expansion and pressure effect in $\mathrm{MnWO}_{4}$
}

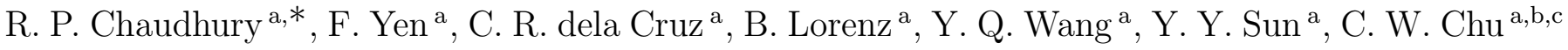 \\ ${ }^{a}$ TCSUH and Department of Physics, University of Houston, Houston, Texas, 77204-5002, USA \\ ${ }^{\mathrm{b}}$ Lawrence Berkeley National Laboratory, 1 Cyclotron Road, Berkeley, California, 94720, USA \\ ${ }^{\mathrm{c}}$ Hong Kong University of Science and Technology, Hong Kong, China
}

Abstract

$\mathrm{MnWO}_{4}$ has attracted attention because of its ferroelectric property induced by frustrated helical spin order. Strong spin-lattice interaction is necessary to explain ferroelectricity associated with this type of magnetic order. We have conducted thermal expansion measurements along the $a, b, c$ axes revealing the existence of strong anisotropic lattice anomalies at $T_{1}=7.8 \mathrm{~K}$, the temperature of the magnetic lock-in transition into a commensurate low-temperature (reentrant paraelectric) phase. The effect of hydrostatic pressure up to $1.8 \mathrm{GPa}$ on the FE phase is investigated by measuring the dielectric constant and the FE polarization. The lowtemperature commensurate and paraelectric phase is stabilized and the stability range of the ferroelectric phase is diminished under pressure.

Key words: $\mathrm{MnWO}_{4}$; thermal expansion; pressure effect; multiferroics PACS: $75.30 .-\mathrm{m}, 75.30 . \mathrm{Kz}, 75.50 . \mathrm{Ee}, 77.80 .-\mathrm{e}, 77.84 . \mathrm{Bw}$

Multiferroic magnetoelectric compounds exhibit the coexistence of ferroelectric (FE) and magnetic orders in some temperature range. The mutual correlation between these orders is of fundamental physical interest and it bears the potential for future applications utilizing the magnetoelectric effect in which the magnetization (FE polarization) is controlled by internal or external electric (magnetic) fields 12. Recently, this property has been observed in $\mathrm{MnWO}_{4}$ in a phase with an incommensurate (IC) helical spin density wave [3. $\mathrm{MnWO}_{4}$ crystalizes in the wolframite structure (monoclinic space group P2/c). Below $15 \mathrm{~K}$ competing magnetic exchange interactions result in a high level of magnetic frustration with several magnetically ordered states quasi-degenerated in energy. As a consequence, $\mathrm{MnWO}_{4}$ undergoes three successive magnetic transitions, antiferromagnetic (AFM) order of the Mn-spins with an IC sinusoidal spin modulation appears at $T_{N}=13.5 \mathrm{~K}$ (AF3 phase) followed by an elliptical IC magnetic order below $T_{2}=12.6$ $\mathrm{K}$ (AF2 phase) and a commensurate collinear magnetic phase below $T_{1}=7.8 \mathrm{~K}$ (AF1 phase) [4]. Ferroelectricity was observed in the AF2 phase only and it can qualitatively be explained by the loss of inversion symmetry due to the helical magnetic order and a strong spin-lattice coupling [5].

\footnotetext{
* Corresponding author. Tel: (713) 743-8314 fax: (713) 743-8201 Email address: rajit.chaudhury@uh.edu (R. P. Chaudhury).
}

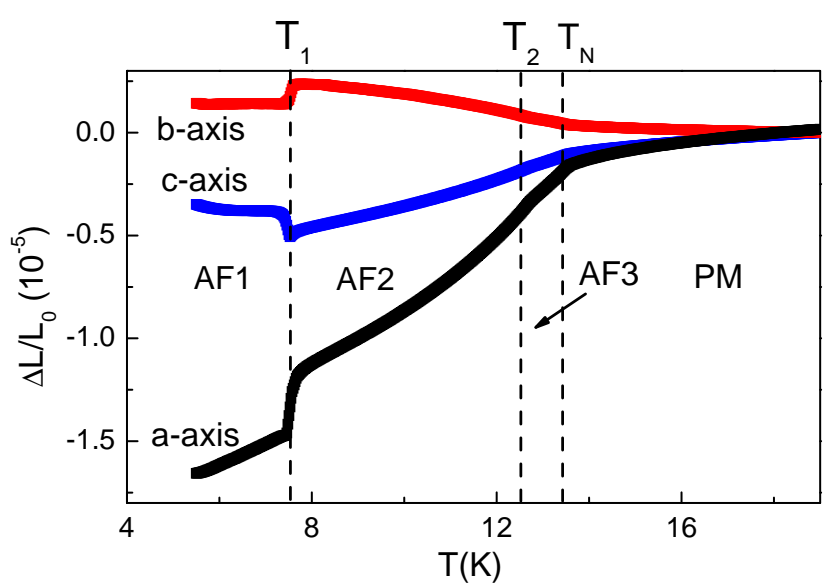

Fig. 1. Thermal expansion of the lattice parameters of $\mathrm{MnWO}_{4}$.

The magnetic phase transitions are also visible in anomalies of the specific heat, the dielectric constant, and the magnetic susceptibility 6].

The coupling between AFM and FE orders observed in $\mathrm{MnWO}_{4}$ must be mediated by strong spin-lattice interactions. The existence of such spin-lattice coupling can be experimentally proven by detecting the strain of the lattice by high-resolution thermal expansion measurements [78]. The macroscopic lattice strain along the principal crystallographic orientations, $a, b$, and $c$, is measured employing a high-resolution capacitance dilatometer. The results shown 


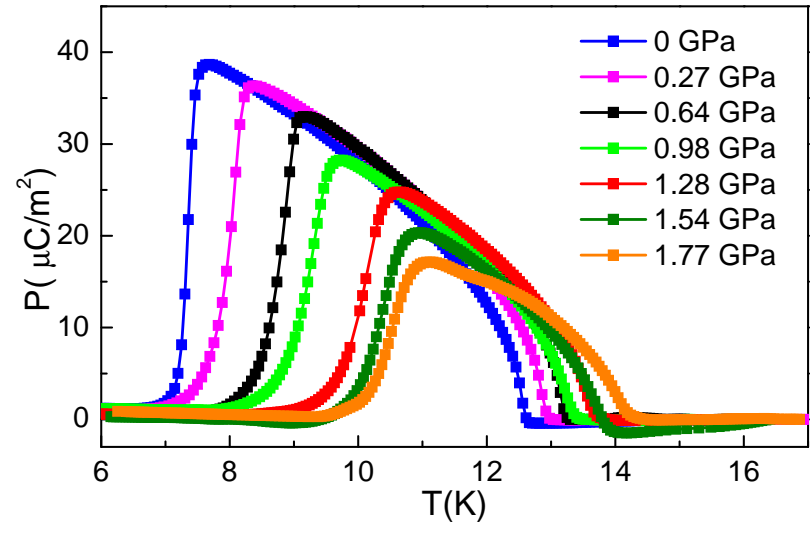

Fig. 2. FE polarization of $\mathrm{MnWO}_{4}$ at various pressures.

in Fig.1 reveal clear anomalies of all three lattice constants at $T_{N}$ (change of slope of all lattice parameters) and at $T_{1}$, the transition into the re-entrant paraelectric state (sharp step-like changes of $a, b, c)$.

Is is important to notice the strong anisotropy of the lattice strain at $T_{1}$. The relative changes of $a, b, c$ and the volume $V$ at $T_{1}$ are: $\Delta a / a=-3 \times 10^{-5}, \Delta b / b=-1 \times 10^{-5}$, $\Delta c / c=1 \times 10^{-5}$ and $\Delta V / V=-3 \times 10^{-5}$, where $\Delta V=$ $V\left(T<T_{1}\right)-V\left(T>T_{1}\right)$. The volume of the AF1 phase is smaller than that of the AF2 phase. The discontinuous volume change across $T_{1}$ proves the first order nature of this phase transition. The anisotropic strain observed in the thermal expansion measurements is associated with the magnetic anisotropy and the peculiar changes of the magnetic order parameter at the various phase transitions. The details of the magnetic structure was revealed in neutron scattering experiments [4. In the collinear AF1 and AF3 phases the spins are aligned with the easy axis of magnetization that lies in the $a-c$ plane at an angle of $37^{\circ}$ with the $a$-axis. In the helical AF2 phase the spin has a component along $b$. The propagation vector $\vec{q}=(-0.214,0.5,0.457)$ of the AF2 and AF3 phases abruptly changes at $T_{1}$ to the commensurate $\vec{q}=(-0.25,0.5,0.5)$ in the AF1 phase. The re-alignment of the spins with the easy axis and the sudden locking of the magnetic modulation with the lattice causes the large decrease of the $a$-axis and the associated volume change. The magnetization along the easy axis also shows the largest change at $T_{1}[6]$.

The strong magnetoelastic effects demonstrated above (Fig. 1) imply a large sensitivity of the AFM and FE orders with respect to lattice strain affecting the interatomic distances and the magnetic exchange interaction parameters. Therefore, external pressure, $p$, can be used to modify the intrinsic magnetic interactions. This is complimentary to the application of external magnetic fields that directly couple to the spins. Of particular interest is the stability of the ferroelectric phase (AF2) with a spontaneous polarization that can be measured by the pyroelectric current method. Fig. 2 shows the FE polarization at different hydrostatic pressures up to $1.8 \mathrm{GPa}$. Both $T_{1}$ and $T_{2}$ increase with $p$ but $T_{1}$ increases at a faster rate diminishing the stability range of the FE phase at higher $p$. The FE polarization is sup-

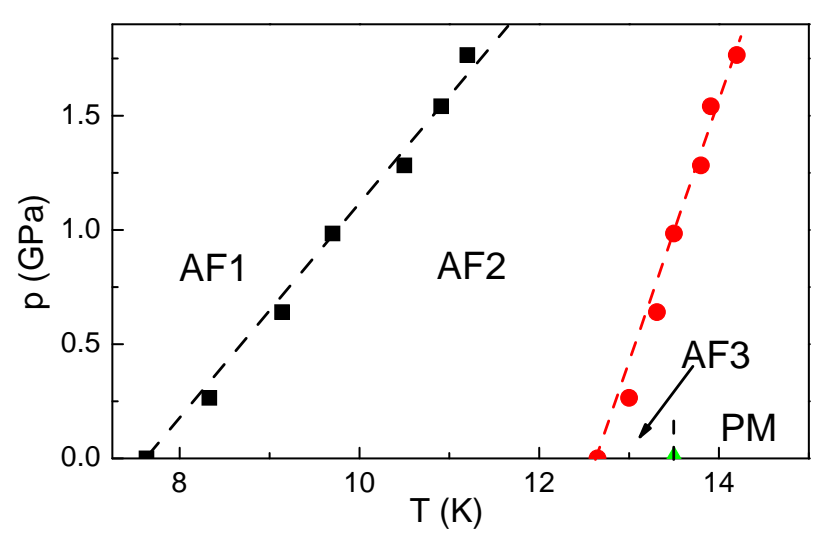

Fig. 3. Pressure-temperature phase diagram of $\mathrm{MnWO}_{4}$.

pressed by pressure, in particular at the low-temperature end of the FE phase. The resulting phase diagram is shown in Fig. 3. The critical temperature of the AF3 phase, $T_{N}$, could not be resolved under pressure. Extrapolating the $p$-dependence of $T_{1}$ and $T_{2}$ we determine the critical pressure above which the FE state becomes unstable as $p_{c} \approx$ $4 \mathrm{GPa}$. The strong increase of $T_{1}$ can be explained by the volume effect. Since the commensurate AF1 phase has a sizably smaller volume than the FE AF2 phase pressure will stabilize the AF1 phase and increase $T_{1}$. The current phase diagram can be compared to the $p$ - $T$ phase diagram of $\mathrm{Ni}_{3} \mathrm{~V}_{2} \mathrm{O}_{8}$ that shows a similar sequence of magnetic and $\mathrm{FE}$ transitions below $10 \mathrm{~K}[8$. In the latter case the critical pressure suppressing ferroelectricity was found to be much lower, $1.64 \mathrm{GPa}$. $\mathrm{Ni}_{3} \mathrm{~V}_{2} \mathrm{O}_{8}$ is very different in terms of lattice structure and symmetry. Unlike $\mathrm{MnWO}_{4}$, the lattice of $\mathrm{Ni}_{3} \mathrm{~V}_{2} \mathrm{O}_{8}$ is highly anisotropic and the magnetic frustration is not only due to competing exchange interactions but also due to the geometry of the Kagome staircase structure of the magnetic Ni-sublattice. This could be the origin for the substantially higher sensitivity of the helical spin structure to the applied pressure.

This work is supported in part by the T.L.L. Temple Foundation, the J. J. and R. Moores Endowment, and the State of Texas through TCSUH and at LBNL through the US DOE.

\section{References}

[1] T. Kimura, T. Goto, H. Shintani, K. Ishizaka, T. Arima, and Y. Tokura, Nature (London) 426, 55 (2003).

[2] T. Goto, T. Kimura, G. Lawes, A. P. Ramirez, and Y. Tokura, Phys. Rev. Lett. 92, 257201 (2004).

[3] K. Taniguchi, N. Abe, T. Takenobu, Y. Iwasa, and T. Arima, Phys. Rev. Lett. 97, 097203 (2006).

[4] G. Lautenschläger, H. Weitzel, T. Vogt, R. Hock, A. Bohm, M. Bonnet, and H. Fuess, Phys. Rev. B 48, 6087 (1993).

[5] M. Mostovoy, Phys. Rev. Lett. 96, 067601 (2006).

[6] A. H. Arkenbout, T. T. M. Palstra, T. Siegrist, and T. Kimura, Phys. Rev. B 74, 184431 (2006).

[7] C. R. dela Cruz, F. Yen, B. Lorenz, M. M. Gospodinov, C. W. Chu, W. Ratcliff, J. W. Lynn, S. Park, and S.-W. Cheong, Phys. Rev. B 73, 100406(R) (2006). 
[8] R. P. Chaudhury, F. Yen, C. R. dela Cruz, B. Lorenz, Y. Q. Wang, Y. Y. Sun, and C. W. Chu, Phys. Rev. B 75, 012407 (2007). 